$63^{\text {ème }}$ Congrès de la SFCO, 03012 (2015)

DOI:10.1051/sfco/20156303012

(C) Owned by the authors, published by EDP Sciences, 2015

\title{
Osteonecrose mandibulaire sous denosumab et anastrozole
}

\author{
Potier J, Grenier S, Cervellera C, Lefevre B \\ Service d'Odontologie, Unité de Chirurgie Orale, Hôpital Maison Blanche, CHU Reims, \\ 45, rue Cognac Jay, 51092 Reims
}

L’ostéonécrose des mâchoires (ONM) est une complication émergente de certains anticorps monoclonaux.

Le cas présenté rapporte l'apparition d'une ONM sous dénosumab et anastrozole.

Une femme de 58 ans est adressée pour l'apparition d'une ulcération gingivale ne cicatrisant pas depuis 3 mois, associée à des algies mandibulaires diffuses et invalidantes qui ont nécessité l'introduction d'un traitement par morphiniques.

Ses antécédents sontun carcinome lobulaire infiltrant du sein gauche, de grade intermédiaire, (récepteurs aux oestrogènes, à la progestérone et Her2 positifs) diagnostiqué deux ans auparavant. Le bilan d'extension (scintigraphie osseuse et IRM cervico-dorso-lombaire) avait mis en évidence la présence de localisations secondaires osseuses rachidiennes pluri-étagées à l'origine de rachialgies. Le traitement a été initialement chirurgical (tumorectomie et curage axillaire gauche), puis une chimiothérapie adjuvante séquentielle, une radiothérapie locale et une hormonothérapie (anastrozole) ont été instaurés.

Un traitement par dénosumab (XGEVA ${ }^{\circledR}, 1$ injection toutes les 4 semaines) avait été instauré un an auparavant, en prévention des complications liées aux métastases osseuses (fractures pathologiques, compression médullaire....).

L'examen endobuccal met en évidence une exposition osseuse en lingual de la 47. La perte de substance mesure $5 \mathrm{~mm} \times 2 \mathrm{~mm}$. Il n'y a pas eu d'acte de chirurgie orale, de soin buccodentaire récent ou de réhabilitation prothétique.

Le cliché panoramique objective de larges plages radio-claires de déminéralisation de la trame osseuse en regard des branches horizontale et montante gauche, le même aspect radiologique est observé dans la région angulaire droite de manière plus localisée. Le bilan est complété par un cone-beam, mettant en évidence des plages hypodensesintraspongieuses multiples, prédominant dans la région angulomandibulaire droite en faveur d'une ostéonécrose.

Le traitement a consisté en la mise en place d'une antibiothérapie par amoxicilline-acide clavulanique 3 grammes par jour pendant 21 jours, des soins locaux et une surveillance clinico-radiologique semestrielle.

Le dénosumab est un anticorps monoclonal IgG2 humain, utilisé couramment en oncologie dans le cadre de la prévention des complications osseuses (compression médullaire, fracture pathologique...) chez les patients atteints de tumeurs solides compliquées de métastases osseuses et en rhumatologie dans le cadre de la prévention de l'ostéoporose. 
Le dénosumab se fixe au RANKL (Receptor-Activatednuclear factor Kappa-B Ligand) avec une affinité et une spécificité élevées, entraînant un blocage de l'interaction RANKL/ récepteur RANK à l'origine d'une réduction du nombre et une inhibition de la fonction des ostéoclastes (résorption osseuse) diminuant ainsi le relargage systémique du calcium osseux (E. Dubois 2011).

Les étiologies de l'ONM ne sont pas clairement définies, mais elles pourraient être liées à l'inhibition du remodelage osseux.

Parmi les patients atteints de cancers exposés audénosumab, le risque d'ONM des mâchoires varie de 0,7 à 1,9 \% (soit 70 à 90 cas pour 10000 patients) (American Association of Oral and Maxillofacial Surgeons, 2014).

L'originalité de ce cas clinique, concerne l'apparition d'une ONM, sans facteur déclenchant. Aucun cas d'ostéonécrose spontanée, sans acte de chirurgie orale, n’a été décrit dans la littérature.

L'association de l'anastrozole au dénosumab pourrait avoir accentué le risque d'ostéochimionécrose par inhibition de l'aromatase, à l'origine d'une carence oestrogénique, avec pour conséquence une réduction de la densité minérale osseuse.

Nom et adresse du conférencier

Julien POTIER

Service d’Odontologie, Unité de Chirurgie Orale, Hôpital Maison Blanche, CHU Reims

45, rue Cognac Jay

51092 Reims (France)

julien.potier-2@hotmail.com 